they can go to a vote in the full Senate and House. Both bills require maintenance of a sharps-injury log and emphasize training, education, and the participation of workers at risk for sharps injuries in evaluating and selecting safer devices. In March 2000, the CDC estimated that 384,325 percutaneous injuries from contaminated sharps occur annually among healthcare workers in the United States.

\section{Skin Tolerance and Effectiveness of Two Hand-Decontamination Procedures}

Hand decontamination is crucial to control nosocomial infections. The utility of hand decontamination is related not only to its antimicrobial effectiveness but also to its acceptability by hospital staff. Winnefeld and colleagues from Marseille, France, conducted a study to assess skin tolerance and antimicrobial effects of two widely accepted hand-hygiene measures under in-use conditions. Fifty-two nurses were randomly assigned for an 8-day period to either an alcohol-based disinfectant or a hand wash with a non-antiseptic soap. At baseline and at the end of the test period, microbiological hand samples were obtained both before and after a hand-hygiene procedure, and skin tolerance was assessed using clinical scores and measurement of transepidermal water loss.

Self-assessment of skin condition and grade of skin damage worsened significantly more in the group using soap than in the group using alcoholic disinfectant $(P=.004$ and $P=.01$, respectively). The alcohol-based rinse was significantly more effective than liquid soap in removing transient contaminant microorganisms $(P=.016)$. Twenty of 50 hand washes with non-antiseptic soap apparently resulted in bacterial contamination of the hands. At the end of the study, the total bacterial count increased with the increasing number of hand washes in the soap group $(P=.003)$ and with the degree of skin damage $(P=.005)$ in the antiseptic group.

The authors concluded that, in everyday hospital practice, alcohol-based disinfectant is more effective and better tolerated than non-antiseptic soap; soap is at risk of spreading contamination; and skin comfort strongly influences the number and the quality of hand-hygiene procedures.

FROM: Winnefeld M, Richard MA, Drancourt M, Grob JJ. Skin tolerance and effectiveness of two hand decontamination procedures in everyday hospital use. $\mathrm{BrJ}$ Dermatol 2000;143:546-550.

\section{Endemic $P$ aeruginosa Infection in an NICU}

Nosocomial infections due to Pseudomonas aeruginosa have been well described, but the environmental reservoir of the organism varies. Foca and coinvestigators from New York-Presbyterian Hospital and Columbia-Presbyterian Medical Center, New York, conducted an epidemiological and molecular investigation of endemic $P$ aeruginos $a$ infection among infants in a neonatal intensive care unit (NICU) that was associated with carriage of the organisms on the hands of healthcare workers (HCWs). In August 1998, col- onization or infection with $P$ aeruginosa was identified in 6 infants. Surveillance cultures were obtained from the other 27 infants in the unit, and possible environmental reservoirs also were assessed. The hands of HCWs were inspected, cultures were taken, and risk factors for $P$ aeruginosa colonization were evaluated. Isolates were analyzed for clonality by pulsed-field gel electrophoresis.

Surveillance cultures showed that 3 additional infants were colonized with Paeruginosa. Cultures of environmental specimens were negative, but cultures of the hands of 10 (6\%) of $165 \mathrm{HCW}$ sere positive for $P$ aeruginosa. Increasing age $(P=.05)$ and a history of the use of artificial fingernails or nail wraps $(P=.03)$ were both risk factors for colonization of the hands. From January 1997 to August 1998, 49 infants were infected or colonized with $P$ aeruginosa. Pulsed-field gel electrophoresis demonstrated that 17 of these infants and $1 \mathrm{HCW}$ who had onychomycosis had the same clone. Infants who were exposed to this HCW in August 1998 were at greater risk of having this clone than infants who were not exposed to this HCW (odds ratio, 41.2; 95\% confidence interval, $1.8-940.0 ; P=.006)$.

The authors concluded that an increased rate of infection and colonization with $P$ aeruginosa among infants in NICUs should be investigated by assessing potential reservoirs, including environmental sources, as well as patients and HCWs.

FROM: Foca M, Jakob K, Whittier S, Della Latta P, Factor S, Rubenstein D, et al. Endemic Pseudomonas aeruginosa infection in a neonatal intensive care unit. $N$ Engl J Med 2000;343:695-700.

\section{Antimicrobial Resistance of $S$ pneumoniae}

Among antimicrobial agents that have been consistently efficacious in treating infections due to specific bacteria over extended periods of time, there are few better examples than Streptococcus pneumoniae and penicillin. Until recently in the United States, this combination had remained nearly uniformly effective. The sole issue mitigating for or against use of penicillin (or ampicillin) in the management of systemic pneumococcal infections or oral ampicillin (or amoxicillin) in treating localized, non-life-threatening pneumococcal infections was the penicillin allergy status of the patient. In the nonallergic patient, penicillin or its congeners have been the drugs of choice largely because resistance to these agents remained uncommon. All of that changed dramatically in the United States during the early part of the 1990 s with the emergence of high rates of antimicrobial resistance with $S$ pneumoniae and concomitantly the recognition of diminished efficacy when certain other antimicrobials were used to treat pneumococcal infections. A recent issue of Seminars in Respiratory and Critical Care Medicine included a thorough discussion of the problem of antimicrobial resistance with $S$ pneumoniae. The discussion, edited by Dr. Gary Doern of the University of Iowa Hospitals and Clinics, includes a question and answer format.

FROM: Doern GV. Antimicrobial resistance with Streptococcus pneumoniae in the United States. Sem Respir Crit Care Med 2000;21:273-284. 